\title{
Postmortem MR quantification of the heart for characterization and differentiation of ischaemic myocardial lesions
}

\author{
Wolf-Dieter Zech • Nicole Schwendener • \\ Anders Persson • Marcel J. Warntjes • \\ Christian Jackowski
}

Received: 16 June 2014 /Revised: 1 October 2014 / Accepted: 19 December 2014 / Published online: 16 January 2015

(C) European Society of Radiology 2015

\begin{abstract}
Objectives Recently, an MRI quantification sequence has been developed which can be used to acquire T1- and T2relaxation times as well as proton density (PD) values. Those three quantitative values can be used to describe soft tissue in an objective manner. The purpose of this study was to investigate the applicability of quantitative cardiac MRI for characterization and differentiation of ischaemic myocardial lesions of different age.

Materials and methods Fifty post-mortem short axis cardiac 3 T MR examinations have been quantified using a quantification sequence. Myocardial lesions were identified according to histology and appearance in MRI images. Ischaemic lesions were assessed for mean T1-, T2- and proton density values. Quantitative values were plotted in a 3D-coordinate system to investigate the clustering of ischaemic myocardial lesions. Results A total of 16 myocardial lesions detected in MRI images were histologically characterized as acute lesions $(n=8)$ with perifocal oedema $(n=8)$, subacute lesions $(n=6)$ and chronic lesions $(n=2)$. In a 3D plot comprising the combined quantitative values of $\mathrm{T} 1, \mathrm{~T} 2$ and $\mathrm{PD}$, the clusters of all investigated lesions could be well differentiated from each other.

Conclusion Post-mortem quantitative cardiac MRI is feasible for characterization and discrimination of different age stages of myocardial infarction.

Key Points

- MR quantification is feasible for characterization of different stages of myocardial infarction.
\end{abstract}

W.-D. Zech $(\bowtie) \cdot$ N. Schwendener $\cdot$ C. Jackowski Institute of Forensic Medicine, University of Bern, Buehlstrasse 20, 3012 Bern, Switzerland

e-mail: Wolf-Dieter.Zech@irm.unibe.ch

A. Persson - M. J. Warntjes

Center for Medical Image Science and Visualization (CMIV),

University of Linköping, 58185 Linköping, Sweden
- The results provide the base for computer-aided MRI cardiac infarction diagnosis.

- Diagnostic criteria may also be applied for living patients.

Keywords Post-mortem quantitative magnetic resonance imaging - Myocardial infarction - Ischaemia - Left ventricular Post-mortem cardiac imaging

$\begin{array}{ll}\text { Abbreviations and Acronyms } \\ \text { MRI } & \text { magnetic resonance imaging } \\ \text { pm MRI } & \text { post mortem resonance imaging } \\ \text { T } & \text { Tesla } \\ \text { T2w } & \text { T2-weighted } \\ \text { PD } & \text { proton density } \\ \text { TR } & \text { repetition time } \\ \text { TE } & \text { echo time } \\ \text { ROI } & \text { region of interest }\end{array}$

\section{Introduction}

Magnetic resonance imaging (MRI) has become a useful tool in post-mortem soft tissue analysis [1-5]. Soft tissue pathology can be conducted in cross sections thinner than possible in any routine autopsy [6-12]. However, MR imaging of a deceased body is very different to clinical MRI, and interpreting post-mortem unenhanced cardiac MR images requires special expertise [13-15]. Cross-sectional postmortem MRI (pm MRI) is used at forensic institutes to assess cause of death as a supplement or adjunct to conventional forensic autopsy $[4,15]$. As cardiac-related deaths represent the major portion of natural deaths, it is of importance that $\mathrm{pm}$ MRI can visualize pathological findings in the human heart [16]. Recent studies visualized myocardial infarction 
performed on pm MRI 1.5-Tesla (T) and 3-T systems. Acute, subacute and chronic infarction were differentiated using the signal behaviour on T1- and T2-weighted (T2w) images [10-15]. To date, the interpretation of pm MRI cardiac images was based on different contrasts at different imaging parameter settings. A more objective approach for differentiation of myocardial infarction in MRI images may be provided by a recently implemented MR sequence that allows for a rapid quantification of relaxation times and proton density (PD). The used sequence is a dual-echo saturation recovery turbo spin-echo acquisition, adapted from the original multi-echo sequence described in Warntjes et al. [18]. This multi-slice technique probes the MR signal intensity after various saturation delays at different echo times (TE), thereby detecting T1 and $\mathrm{T} 2$ relaxation times simultaneously. When $\mathrm{T} 1$ and $\mathrm{T} 2$ relaxation times (TR) are determined, the combination allows for a calculation of the absolute signal intensity at $\mathrm{TE}=0 \mathrm{~ms}$ and infinite saturation delay time, equal to PD. T1 and T2 relaxation times as well as PD can be measured in a single acquisition. Based on the relaxation times and PD any conventional tissue contrast image, with a certain combination of TE and TR or prepulses, may be reconstructed using an additional post-processing step called synthetic MRI [17-21].

Zech et al. [33] previously showed that in a post-mortem MRI application the quantified parameters T1, T2 and PD can be used to characterize and differentiate thoraco-abdominal organs and soft tissues in an objective manner independently of the image reader. Warntjes et al. [18] demonstrated that it is possible to characterize inflammatory lesions in the brains of MS patients in vivo using quantitative brain MRI. It can be assumed that the cardiac MRI quantification approach can be used to characterize and differentiate ischaemic myocardial lesions. Thus, the purpose of the present study was to investigate the feasibility of quantitative cardiac pm MRI for age characterization and differentiation of ischaemic myocardial lesions.

\section{Materials and methods}

\section{Study population}

In forensic cases ( $\mathrm{n}=50 ; 14$ female, 36 male) a quantification sequence was added to a post-mortem cardiac 3 T MR examination (Achiva, Philips Healthcare, best, The Netherlands). The examinations were performed at the Institute of Forensic Medicine, University of Zürich, Switzerland. All cases presented either with a known history of myocardial infarction or with an acute cardiac anamnesis prior to death. Age at death ranged from 11 to 94 years (mean 52 years, SD 18.3;). In all cases the local authorities ordered a forensic autopsy and the MR examination was performed prior to autopsy. The post- mortem interval (time from death to scanning) ranged from several hours to 3 days.

\section{Magnetic resonance imaging}

Relevant putrefaction gas formation was ruled out using whole body computed tomography prior to pm MRI. The corpses were wrapped either in an artefact-free body bag, a linen sheet or a plastic foil. All subjects were examined in the supine position. MR examination time was $2 \mathrm{~h}$, of which the quantification sequence lasted $15 \mathrm{~min}$. Twenty to 24 short axis slices of $4 \mathrm{~mm}$ thickness were acquired with a gap of $0.3 \mathrm{~mm}$. Use of the image data for the present study was approved by the local ethics committee. Using a commercially available post-processing tool (SyMRI Autopsy, SyntheticMR, Linköping, Sweden) [22] T1- and T2 relaxation times and $\mathrm{PD}$ of normal myocardium in cardiac short axis images were assessed by placing five regions of interest (ROIs) in lesionfree regions. Lesions of sufficient size allowing placing five different and independent ROIs within one single lesion were additionally quantified and included in the study (Fig. 1). The mean $\mathrm{T} 1$ and $\mathrm{T} 2$ relaxation times in ms as well as PD (\%) were plotted in a 3D plot using the ThreeDifyExcelGrapher Add in for Microsoft Excel ${ }^{\circledR}$ to investigate clustering and separation of myocardial lesions of different age.

\section{Morphological correlation}

MR image findings of myocardial lesions were correlated with autopsy and histology findings in terms of location and age assessment by board-certified forensic pathologists. Myocardial lesions were classified as acute, subacute and chronic infarction/scar. Perifocal myocardial oedema adjacent to acute lesions was assessed as well. Pathological myocardial lesions were diagnosed according to the following criteria of Jackowski et al. [15]: Acute lesions: autopsy aspect: yellowish discoloration; histology: fibre necrosis, granulocytous infiltration, myocardial oedema. Subacute lesions: autopsy aspect: greyish myocardial alteration with slightly denser consistency; histology: fibroblasts with loose connective tissue formation, angiogenesis. Chronic lesions: autopsy aspect: white shrunken left ventricular wall; histology: nuclei-free collagenous fibres. Elucidated and disaggregated myocardial tissue adjacent to acute myocardial lesions was classified as perifocal oedema. Board-certified forensic pathologists that were blinded for the MRI findings carried out the autopsy directly after imaging or the following morning. Cardiac dissection was performed by base parallel slicing of the heart in order to match short axis images on pm MRI. All lesions detected in pm MRI were examined histologically. Histology stains included haematoxylin and eosin, elastic van Gieson and chromotrop-aniline-blue. 
Fig. 1 Cardiac short axis synthetically calculated T2w MRI image. Note an acute myocardial lesion with perifocal oedema within the lateral left ventricular region. Quantitative values for this lesion (red frame upper right corner) were obtained by placing the ROI into the lesion

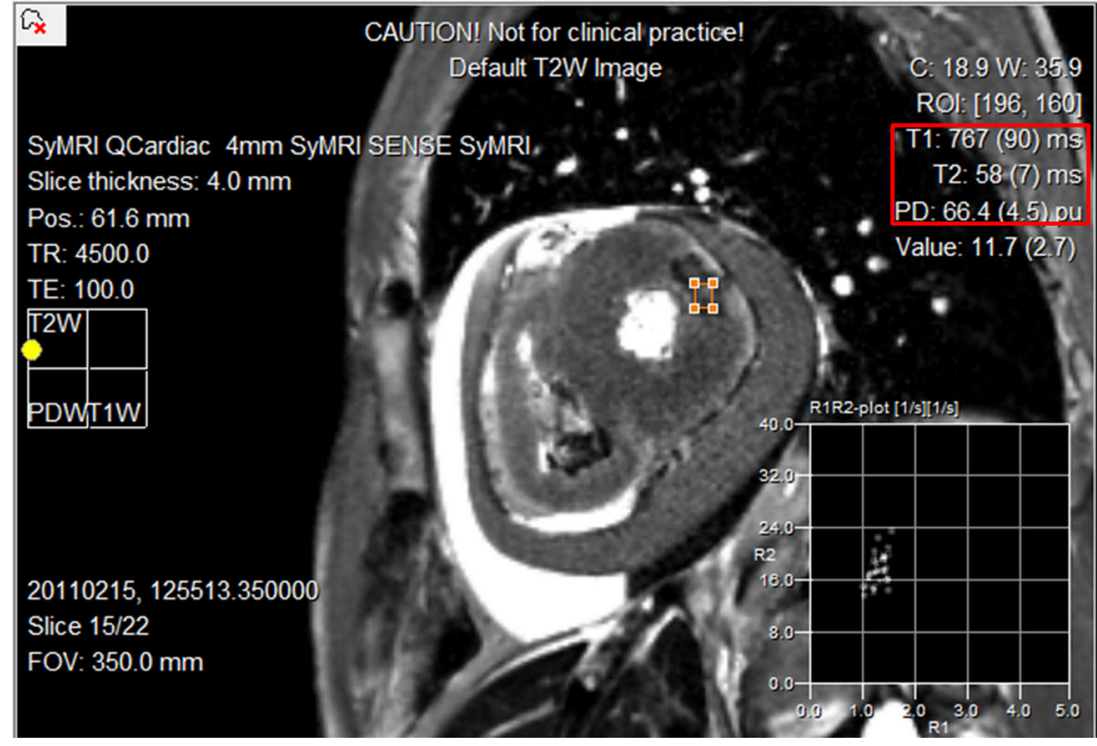

Statistics

A two-sided paired t-test with assumed equal variances was carried out to observe for significant differences of the quantitative mean T1, T2 and PD values between all myocardial lesions and normal myocardium. Furthermore, t-testing was carried out for correlation of relaxation times and PD of healthy/diseased myocardium and post-mortem interval as well as for correlation of relaxation times and PD of healthy/ diseased myocardium and the age of the deceased persons.

The null hypothesis was rejected when the p-value was less than 0.05 .

\section{Results}

From 50 forensic cases a total of 16 (13 male, three female, mean age 62.20 years, SD 20.41) displayed visible tissue alterations of sufficient size in cardiac pm MRI images to be included. Those lesions were histologically characterized as acute lesions $(n=8)$, subacute lesions $(n=6)$ and chronic lesions / scars $(n=2)$. All cases with acute lesions presented with perifocal oedema $(n=8)$ (Fig. 1). Due to small case numbers chronic lesions were removed. In the 34 persons that did not display visible lesions in the MRI images there were also no detectable acute or subacute lesions in histological examinations.

Figure 2 depicts boxplots of the mean quantitative values (out of five ROI measurements) of T1, T2 and PD obtained for myocardial lesions and normal myocardium. Quantitative values were plotted in a 3D-coordinate System. In the 3Dplot clusters of acute lesions, subacute lesions and perifocal oedema could be well differentiated from each other (Fig. 3). A significant difference between the quantitative values of all myocardial lesions and normal myocardium could be observed in the t-test (Table 1). Slightly overlapping clusters were observed for normal myocardium and acute lesions. However, differentiation between normal myocardium and acute myocardial lesions became indirectly possible by assessing myocardial oedema that was found to be surrounding acute lesions only.

T-testing revealed no correlation between the quantitative values for T1, T2 and PD compared to the age of the deceased or the post-mortem interval.

\section{Discussion}

Quantitative MRI sequences allow for simultaneous determination of T1 and T2 tissue relaxation times as well as for tissue PD [17-20]. In the present post-mortem study, this MRI approach was applied for the first time to investigate ischaemic myocardial lesions. The results indicate that myocardial lesions of different age can be differentiated from each other by their quantitative values of T1, T2 and PD. This provides the base for additional promising applications of quantitative cardiac MRI. Databases for quantitative values of T1, T2 and PD of normal myocardium as well as of altered myocardium can be established. As a result, a scale of quantitative values of regular myocardial tissue and pathological myocardial lesions can be developed [21]. Using quantitative data from synthetic cardiac MRI applications also provides the basis for automatic detection of myocardial lesions. Synthetic cardiac MR images can be loaded on a personal computer with recently developed software. Computer-aided diagnostics can be implemented based on established ranges of the signal behaviour of normal myocardium and pathological lesions [21, 23, 24]. Myocardial lesions can, for example, automatically be detected and encoded in different colours. This can help the image reader 
Fig. 2 Boxplots of T1, T2 and PD quantification values (mean of five measurements) of normal myocardium as well as acute and subacute ischaemic myocardial lesions

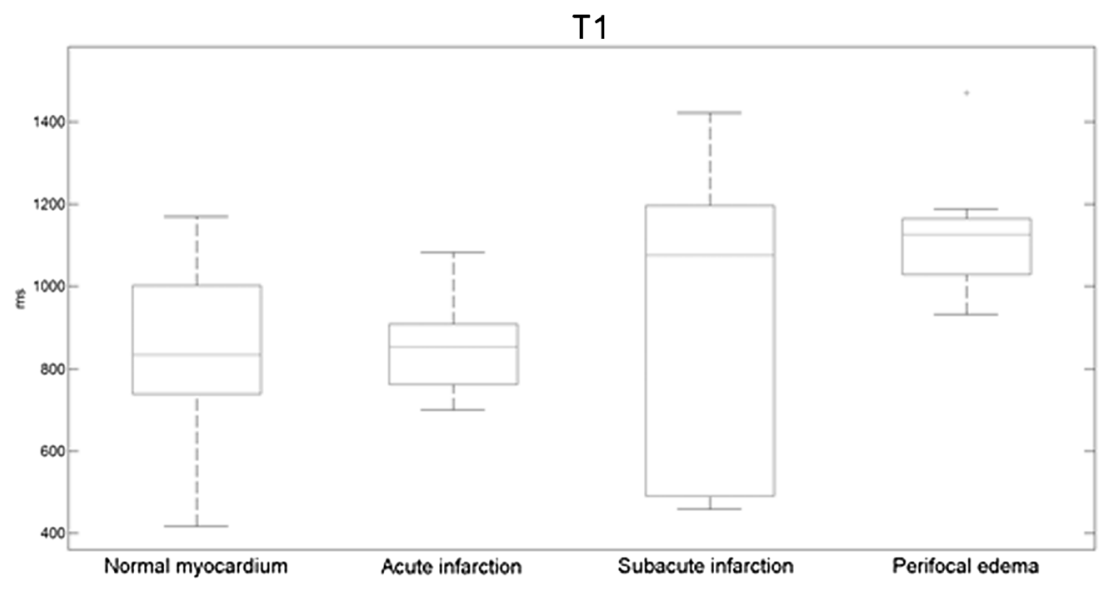

T2

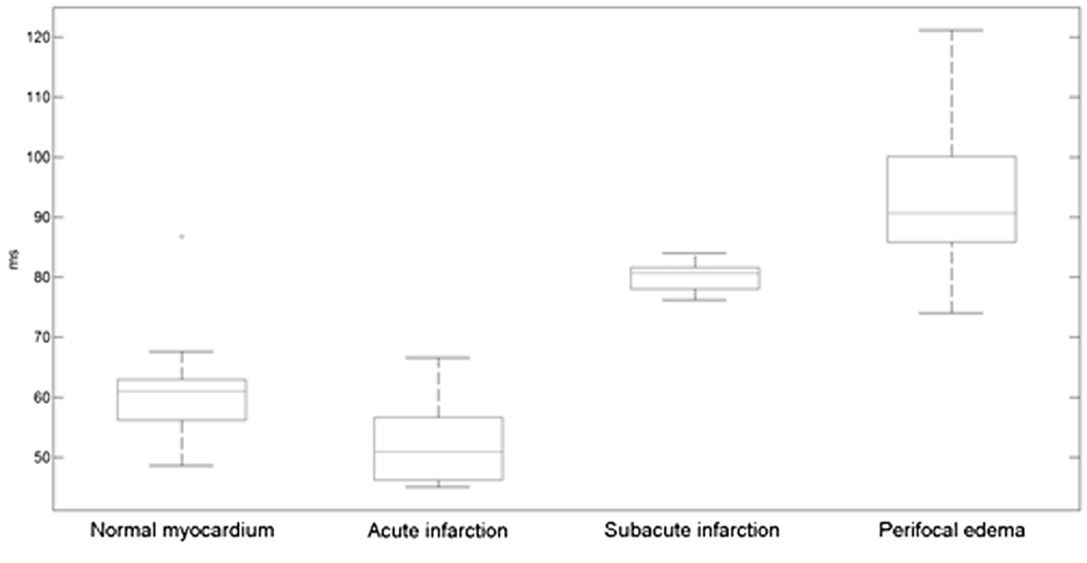

PD

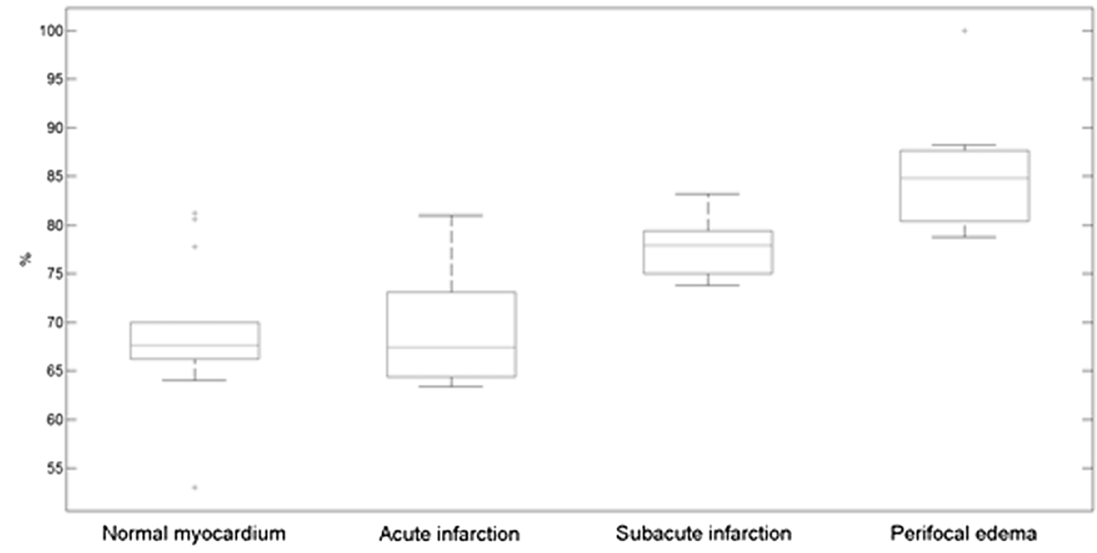

to detect myocardial lesions. Moreover, information regarding the nature and age of the lesion can be obtained. It can be assumed that quantitative cardiac MRI also has the potential to detect lesions neither visible in histology nor in conventional cardiac MRI imaging. Warntjes et al. [18] demonstrated in vivo that using synthetic MRI inflammatory multiple sclerosis lesions in the brain can be differentiated from normal healthy brain tissue by their quantitative T1, T2 and PD values. Hence, synthetic cardiac MRI may also be feasible for detecting lesions other than ischaemic ones, such as myocardial inflammation. Furthermore degenerative processes such as amyloidosis may be detectable [7]. Further studies are required to reveal the factual diagnostic possibilities and limits of synthetic cardiac pm MRI.

Study limitations

The present study has several limitations. Data were obtained from 50 cases of which only 16 presented with myocardial lesions of sufficient size. In order to create a database with 


\section{a}

$$
\mathrm{T} 2 \text { (ms) }
$$

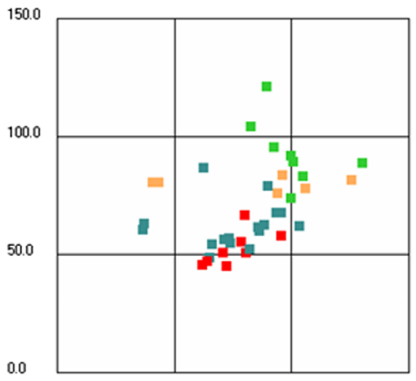

$\mathrm{T} 1$ (ms) b

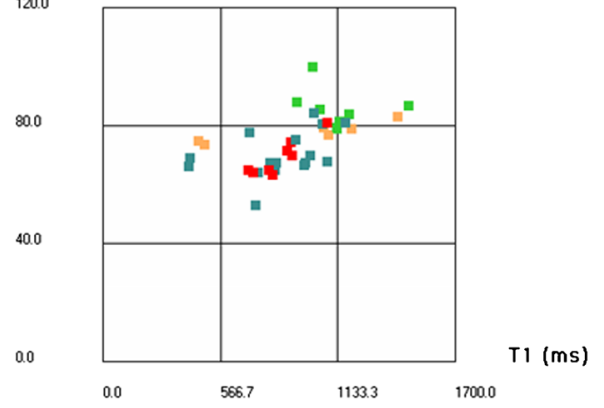

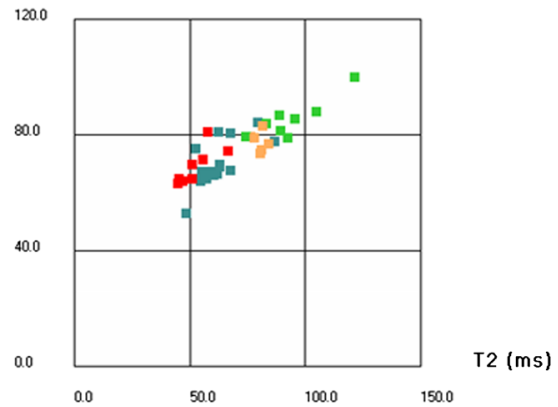

Acute infarction O Normal Myocard O Perifocal edema O Subacute infarction

Fig. 3 Three defined views on a 3D plot of different ischaemic myocardial alterations based on measured relaxation times and proton density. $\mathrm{PD} / \mathrm{T} 2$ view (a), $\mathrm{PD} / \mathrm{T} 1$ view (b) and T2/T1 view (c). Note that

quantitative T1, T2 and PD values for various myocardial lesions higher case numbers are required. Furthermore, the low case numbers did not allow for statistical analysis of data. best discrimination by less overlapping is seen along the $\mathrm{T} 1$ projection within the $\mathrm{PD} / \mathrm{T} 2$ view (a) (values are not corrected for core temperature at acquisition)

Zech et al. [33] recently demonstrated temperature dependence of myocardium mainly for quantitative $\mathrm{T} 1$ values that was increasing with rising temperature. Correction of

Table 1 T-test for comparison of quantitative mean values between myocardial lesions and normal myocardium

\begin{tabular}{|c|c|c|c|c|}
\hline & & $\mathrm{T} 1^{*}$ & $\mathrm{~T} 2^{\dagger}$ & $\mathrm{PD}^{\ddagger}$ \\
\hline \multirow[t]{2}{*}{ Normal myocardium-subacute infarction } & p-value & 0.465595 & 0.000266 & 0.034945 \\
\hline & Significance & no & yes & yes \\
\hline \multirow[t]{2}{*}{ Normal myocardium-acute infarction } & p-value & 0.972563 & 0.020403 & 0.766867 \\
\hline & Significance & no & yes & no \\
\hline \multirow[t]{2}{*}{ Normal myocardium-perifocal oedema } & p-value & 0.004648 & 0.000002 & 0.000114 \\
\hline & Significance & yes & yes & yes \\
\hline \multirow[t]{2}{*}{ Acute infarction-subacute infarction } & p-value & 0.506781 & 0.000002 & 0.010407 \\
\hline & Significance & no & yes & yes \\
\hline \multirow[t]{2}{*}{ Acute infarction-perifocal oedema } & $\mathrm{p}$-value & 0.001645 & 0.000004 & 0.000194 \\
\hline & Significance & yes & yes & yes \\
\hline \multirow[t]{2}{*}{ Perifocal oedema-Ssbacute infarction } & p-value & 0.265556 & 0.044707 & 0.026333 \\
\hline & Significance & no & yes & yes \\
\hline
\end{tabular}

${ }^{*} \mathrm{~T} 1$ in $\mathrm{ms} ;{ }^{\dagger} \mathrm{T} 2$ in $\mathrm{ms} ;{ }^{\star} \mathrm{PD}$ in \%. The t-test was performed separately between $\mathrm{T} 1, \mathrm{~T} 2$ and PD variables. Note in every case (except for normal myocardium compared with acute infarction) there are at least two significantly different centre coordinates. A significant difference between all tested lesions and normal myocardium can be observed 
quantitative values to $37{ }^{\circ} \mathrm{C}$ resulted in a statistically better discrimination between different thoraco-abdominal organs. In the present study no corpse temperatures were assessed. Therefore a temperature correction of quantitative values was not possible. Considering the previous results of Zech et al. [18], temperature correction of quantitative values in the present study may have led to tighter clustering and better differentiation between myocardial lesions.

As temperature dependence most influences $\mathrm{T} 1$ values, the broad ranges of $\mathrm{T} 1$ values in the present study may be explained by different corpse temperatures at imaging. If quantitative data acquired from post-mortem myocardial lesions are corrected to a temperature of $37^{\circ} \mathrm{C}$ they may be comparable to in vivo data. In the current literature only few in vivo quantitative $3 \mathrm{~T}$ MRI studies with T1 and T2 values of healthy myocardium can be found [25-28]. Even without a temperature correction the quantitative $\mathrm{T} 1$ and $\mathrm{T} 2$ values of the present post-mortem MRI study for healthy myocardium can be classified within the range of the quantitative in vivo $\mathrm{T} 1$ and $\mathrm{T} 2$ values for healthy myocardium from literature. There are only very few $3 \mathrm{~T}$ MRI in vivo studies that evaluated the quantitative $\mathrm{T} 1$ and $\mathrm{T} 2$ values of pathological myocardial tissue. Furthermore in these studies only very few patients were examined. However, as in the present post-mortem study, Heeswijk et al. [29] and Blume et al. [30] were able to detect an in vivo increase of the $\mathrm{T} 2$ values in myocardial tissue with oedema and subacute infarction. This indicates that the quantitative values for pathological tissues evaluated from post-mortem cases may be applied for clinical use as well. According to the current literature no in vivo 3 T MRI studies were conducted involving the proton density as a quantitative parameter. Since in the present study PD values increased in myocardial tissue with oedema and subacute infarction the use of the PD for myocardial tissue characterization in vivo may also be promising.

Another limitation of the present study is that peracute myocardial lesions (age between minutes and approximately $1 \mathrm{~h}$ ) were not assessed. Sudden cardiac death, without the finding of a fresh ischaemic myocardial lesion, challenges pathological and forensic examinations since peracute lesions are hardly detectable at autopsy and routine histological examination [11]. However, Jackowski et al. [11, 15] demonstrated that hypointensities on unenhanced conventional $\mathrm{T} 2 \mathrm{w}$ images without any hyperintense margin may be a sign of peracute ischaemic lesions. It is not fully understood why MR seems to be more sensitive to peracute myocardial lesions than conventional histology. Hypotheses include the early drop in $\mathrm{pH}$ value that shortens $\mathrm{T} 2$ relaxation. A possible contribution of decreased interstitial water has also been discussed due to insufficient arterial blood supply while the venous drainage is not affected [31]. It can be concluded that quantitative MRI of the heart assessing values for $\mathrm{T} 2$ relaxation times may therefore also be feasible for detecting peracute lesions in the future.
For the present study a 3 Tesla MRI was used. The relaxation behaviour of tissues is influenced by the magnitude of the magnetic field. Therefore it is to be expected that the use of MRI scanners with different magnetic field strengths, such as 1,5 Tesla, will result in different quantitative values for relaxation times of $\mathrm{T} 1$ and $\mathrm{T} 2$ for the same tissue and temperature [32]. Quantitative values of relaxation times from myocardial tissue have to be adapted to different magnetic field strengths in future studies.

\section{Conclusion}

The results of the presented study indicate that quantitative cardiac pm MRI is feasible for characterizing and discriminating different age stages of myocardial infarction. Thereby, it can support forensic autopsy by allowing for targeted histological examination. By providing the tool for automated tissue detection it may also serve to recognize peracute ischaemic lesions not visible in histology or conventional cardiac MRI images.

Acknowledgements The scientific guarantor of this publication is Prof. Christian Jackowski MD, Head of Departement Institute of Forensic Medicine Bern, Switzerland. The authors of this manuscript declare no relationships with any companies, whose products or services may be related to the subject matter of the article. The authors state that this work has not received any funding. Beat Kneubuehl, $\mathrm{PhD}$, kindly provided statistical advice for this manuscript. Institutional Review Board approval was not required because all cases/corpses were investigated by order of the local prosecutors. The prosecutors agreed to research on corpses when the personal data of the deceased persons are treated strictly confidential. Since we treat all personal data of corpses as confidential we have information from the Institutional Review Board that approval from the Review Board is not necessary for all our conducted post-mortem studies including post-mortem imaging. Written informed consent was not required for this study because the subjects studied were deceased persons. None of the study subjects or cohorts have been previously reported. Methodology: prospective, experimental, performed at one institution.

\section{References}

1. Patriquin L, Kassarjian A, Barish M et al (2001) Postmortem wholebody magnetic resonance imaging as an adjunct to autopsy: preliminary clinical experience. J Magn Reson Imaging 13:277-87

2. Roberts IS, Benbow EW, Bisset R et al (2003) Accuracy of magnetic resonance imaging in determining cause of sudden death in adults: comparison with conventional autopsy. Histopathology 42:424-30

3. Dirnhofer R, Jackowski C, Vock P, Potter K, Thali MJ (2006) VIRTOPSY: minimally invasive, imaging-guided virtual autopsy. Radiographics 26:1305-33

4. Lundström C, Persson A, Ross S et al (2012) State-of-the-art of visualization in post-mortem imaging. APMIS 120:316-26

5. Jackowski C, Thali MJ, Buck U et al (2006) Noninvasive estimation of organ weights by postmortem magnetic resonance imaging and multislice computed tomography. Invest Radiol 41:572-78 
6. Jackowski C, Thali MJ, Aghayev E et al (2006) Postmortem imaging of blood and its characteristics using MSCT and MRI. Int J Legal Med 120(4):233-40

7. Fitzmaurice GJ, Wishart V, Graham AN (2013) An unexpected mortality following cardiac surgery: a post-mortem diagnosis of cardiac amyloidosis. Gen Thorac Cardiovasc Surg 61:417-21

8. Aghayev E, Sonnenschein M, Jackowski C et al (2006) Postmortem radiology of fatal hemorrhage: measurements of cross-sectional areas of major blood vessels and volumes of aorta and spleen on MDCT and volumes of heart chambers on MRI. AJR Am J Roentgenol 187: 209-15

9. Kuroiwa Y, Yamashita A, Nishihira K et al (2011) Cardiac rupture in acute myocardial infarction: post-mortem MR imaging. Magn Reson Med Sci 10:255-58

10. Jackowski C, Christe A, Sonnenschein M, Aghayev E, Thali MJ (2006) Postmortem unenhanced magnetic resonance imaging of myocardial infarction in correlation to histological infarction age characterization. Eur Heart J 27:2459-67

11. Jackowski C, Warntjes MJ, Berge J, Bar W, Persson A (2011) Magnetic resonance imaging goes postmortem: noninvasive detection and assessment of myocardial infarction by postmortem MRI. Eur Radiol 21:70-8

12. Thayyil S, Sebire NJ, Chitty LS et al (2013) Post-mortem MRI versus conventional autopsy in fetuses and children: a prospective validation study. Lancet 382:223-33

13. Jackowski C, Hofmann K, Schwendener N, Schweitzer W, KellerSutter M (2011) Coronary thrombus and peracute myocardial infarction visualized by unenhanced postmortem MRI prior to autopsy. Forensic Sci Int 214:e16-e9

14. Jackowski C, Schweitzer W, Thali MJ et al (2005) Virtopsy: postmortem imaging of the human heart in situ using MSCT and MRI. Forensic Sci Int 149:11-23

15. Jackowski C, Schwendener N, Grabherr S, Persson A (2013) Postmortem cardiac $3 \mathrm{~T}$ magnetic resonance imaging: Visualizing the sudden cardiac death? J Am Coll Cardiol 62:617-29

16. Jachau K, Heinrichs T, Kuchheuser W et al (2004) Computed tomography and magnetic resonance imaging compared to pathoanatomic findings in isolated human autopsy hearts. Rechtsmedizin 14:109-16

17. Warntjes JB, Dahlqvist O, Lundberg P (2007) Novel method for rapid, simultaneous $\mathrm{T} 1, \mathrm{~T} * 2$, and proton density quantification. Magn Reson Med 57:528-37

18. Warntjes JB, Leinhard OD, West J, Lundberg P (2008) Rapid magnetic resonance quantification on the brain: Optimization for clinical usage. Magn Reson Med 60:320-29

19. Warntjes MJ, Kihlberg J, Engvall J (2010) Rapid T1 quantification based on 3D phase sensitive inversion recovery. BMC Med Imaging. doi:10.1186/1471-2342-10-19
20. Blystad I, Warntjes JB, Smedby O et al (2012) Synthetic MRI of the brain in a clinical setting. Acta Radiol 53:1158-63

21. Jackowski C, Warntjes MJ, Kihlberg J, Berge J, Thali MJ, Persson A (2011) Quantitative MRI in isotropic spatial resolution for forensic soft tissue documentation. Why and how? J Forensic Sci 56:208-15

22. SyntheticMR products website. Available at: http://www. syntheticmr.com. Accessed May 31, 2014.

23. Ljung P, Winskog C, Persson A, Lundström C, Ynnerman A (2006) Full body virtual autopsies using a state-of-the-art volume rendering pipeline. IEEE Trans Vis Comput Graph 12:869-76

24. Persson A, Lindblom M, Jackowski C (2011) A state-of-the-art pipeline for postmortem $\mathrm{CT}$ and MRI visualization: from data acquisition to interactive image interpretation at autopsy. Acta Radiol 52: $522-36$

25. H-Ici DO, Jeuthe S, Al-Wakeel N et al (2014) T1 mapping in ischemic heart disease. Eur Heart J Cardiovasc Imaging 15(6):597602

26. Sharma V, Binukrishnan S, Schoepf UJ, Ruzsics B (2014) Myocardial Tissue Characterization With Magnetic Resonance Imaging. J Thorac Imaging Jan 4. [Epub ahead of print]

27. Ding H, Fernandez-de-Manuel L, Schär M et al (2014) Threedimensional whole-heart T2 mapping at 3T. Magn Reson Med. doi: $10.1002 / \mathrm{mrm} .25458$

28. Karmonik C, Malaty A, Bikram M, Schmitt P, Partovi S, Shah DJ (2014) Fast in vivo quantification of T1 and T2 MRI relaxation times in the myocardium based on inversion recovery SSFP with in vitro validation post Gd-based contrast administration. Cardiovasc Diagn Ther 4(2):88-96

29. Van Heeswijk RB, Feliciano H, Bongard C et al (2012) Freebreathing $3 \mathrm{~T}$ magnetic resonance T2-mapping of the heart. JACC Cardiovasc Imaging 5(12):1231-9

30. Blume U, Lockie T, Stehning C et al (2009) Interleaved T(1) and T(2) relaxation time mapping for cardiac applications. J Magn Reson Imaging 29(2):480-7

31. Jansen CH, Perera D, Makowski MR et al (2011) Detection of intracoronary thrombus by magnetic resonance imaging in patients with acute myocardial infarction. Circulation 124:416-24

32. Haacke ME, Brown RW, Thompson MR, Venkatesh N (1999) Magnetic resonance imaging-physical principles and sequence design, 1st edn. John Wiley \& Sons, New York

33. Zech W, Schwendener N, Kneubuehl B, Persson A, Warntjes MJ, Jackowski C. (2014) Temperature dependence of postmortem MR quantification for soft tissue discrimination and sequence optimization. Submitted for publication in European Radiology; April 2014 